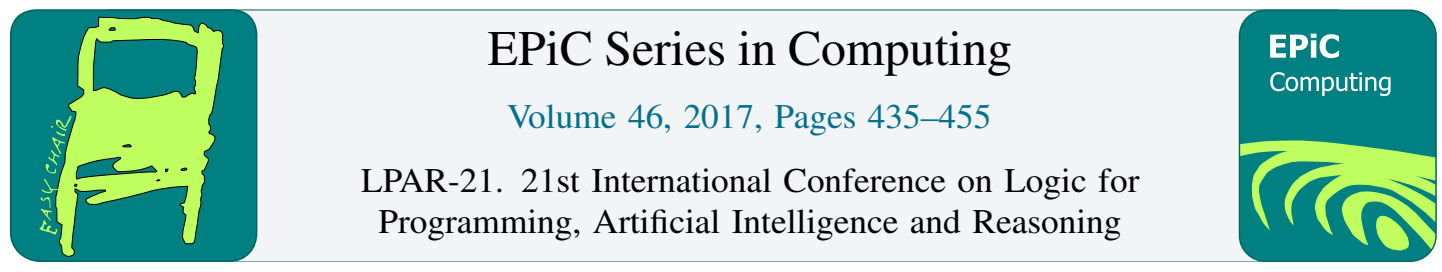

\title{
A uniform framework for substructural logics with modalities
}

\author{
Björn Lellmann ${ }^{1}$, Carlos Olarte ${ }^{2}$ and Elaine Pimentel ${ }^{3}$ * \\ 1 Department of Computer Languages, TU Wien, Austria \\ 2 ECT, UFRN, Brazil \\ 3 Department of Mathematics, UFRN, Brazil
}

\begin{abstract}
It is well known that context dependent logical rules can be problematic both to implement and reason about. This is one of the factors driving the quest for better behaved, i.e., local, logical systems. In this work we investigate such a local system for linear logic (LL) based on linear nested sequents (LNS). Relying on that system, we propose a general framework for modularly describing systems combining, coherently, substructural behaviors inherited from LL with simply dependent multimodalities. This class of systems includes linear, elementary, affine, bounded and subexponential linear logics and extensions of multiplicative additive linear logic (MALL) with normal modalities, as well as general combinations of them. The resulting LNS systems can be adequately encoded into (plain) linear logic, supporting the idea that LL is, in fact, a "universal framework" for the specification of logical systems. From the theoretical point of view, we give a uniform presentation of LL featuring different axioms for its modal operators. From the practical point of view, our results lead to a generic way of constructing theorem provers for different logics, all of them based on the same grounds. This opens the possibility of using the same logical framework for reasoning about all such logical systems.
\end{abstract}

\section{Introduction}

One feature common to most logics with modalities is that the sequent rules for the modal connectives are context dependent. For example, in classical linear logic (LL) [Gir87] the promotion rule

$$
\frac{? \Gamma, F}{? \Gamma, ! F} \text { prom }
$$

is such that the bang ! can be introduced only if the context is classical, i.e., all formulas in $\Gamma$ are marked with ?. This lack of locality is often a problem for (1) describing different modalities in a modular way and (2) proving meta-level properties about the systems, such as cut-elimination.

To tackle the first issue, a number of generalizations of the sequent calculus have been considered, such as labelled sequents [Vig00, NvP11], nested or tree-hypersequent [Brü09, Pog09], and their restriction to 2-sequents or linear nested sequents [Mas92, GMM98, Lel15, LP15]. Here we concentrate on the

\footnotetext{
${ }^{*}$ Pimentel and Olarte are funded by CNPq and CAPES. Lellmann is funded by the European Union's Framework Programme for Research and Innovation Horizon 2020 under Marie Skłodowska-Curie grant agreement No. [660047].
} 
latter approach. Intuitively, a linear nested sequent (LNS) is list of standard sequents separated by the nesting operator //, with the head interpreted in the usual way and the tail interpreted (recursively) under a modal operator. The logical rules then act on the elements of the list, possibly moving formulas from one element to another. This finer way of representing systems enables both locality and modularity by decomposing standard sequent rules into smaller components. Indeed, consider the well known sequent rules $\mathrm{k}$ and $\mathrm{d}$ :

$$
\frac{\Gamma, A}{\diamond \Gamma, \square A, \Delta} \mathrm{k} \quad \frac{\Gamma, A}{\diamond \Gamma, \diamond A, \Delta} \mathrm{d}
$$

Observe that neither of them is local, since both introduce more than one connective at a time. Instead, the modal rule $\mathrm{k}$ in the linear nested setting is decomposed into the two rules

$$
\frac{\mathcal{G} / / \Gamma / / \Delta, A / / \mathcal{H}}{\mathcal{G} / / \Gamma, \diamond A / / \Delta / / \mathcal{H}} \diamond \quad \frac{\mathcal{G} / / \Gamma / / A}{\mathcal{G} / / \Gamma, \square A} \square
$$

Note that different connectives are introduced one at a time by different (context free) rules, and this entails locality. Moreover, decomposing the sequent rules enables modularity since now extensions of, e.g., the modal system $\mathrm{K}$ are obtained by adding the respective (local) modal rules. For instance, to extend the calculus for $\mathrm{K}$ to a calculus for $\mathrm{KD}$, only the rule $\mathrm{d}$ needs to be added:

$$
\frac{\mathcal{G} / / \Gamma / / A}{\mathcal{G} / / \Gamma, \diamond A} \mathrm{~d}
$$

As a welcome side effect, in the LNS systems the modal connectives are canonical, i.e., uniquely defined by the modal rules, a property which is well-known not to hold for most standard sequent representations.

Regarding (2), in a series of works [MP02, MP04, PM05, MP13], linear logic was used as a framework for specifying and reasoning about sequent systems. While LL was shown to be general enough for capturing most classical and intuitionistic features, basically the only modalities that could be specified were its own, i.e., ! and ?. More complex modalities, such as the one of S4, or substructural features, such as multi-conclusion intuitionistic systems, seemed to require more complex mechanisms such as extensions of LL with subexponentials [DJS93] (see [NPR14]). While this could signal that LL is not general enough for capturing certain features of proof systems, it may also indicate that some proof systems are just not adequate for describing modalities. For instance, in [LP15], it was shown that using the decomposition of sequent rules into linear nested sequent rules as described above, LL could be used for specifying sequent systems for various normal modal logics. This entailed two interesting results: modular theorem provers could be automatically generated for such logics; and LL, as a logical framework, could be used for reasoning about object level properties of modal logics.

In this paper we generalize these results to the linear (resource conscious) setting. On understanding better the locality of LL, we are able to modularly describe other kinds of modalities as well as other substructural behaviors. More precisely, it is well-known that the exponential ? of LL has a modal behavior similar to the modal diamond in normal modal logic S4 [MM94]. Further, LL allows both weakening and contraction on question-marked formulas. Interesting questions then are: (a) what kind of resource conscious modal logics arise from changing the modal behavior of ?; (b) what kind of substructural properties for such logics can be captured when restricting weakening and contraction in ?; (c) how can such new modal/substructural systems be combined while preserving properties such as cut-elimination?

Questions (a) and (b) were considered, e.g., in [GMM98, MM94] where 2-sequents were used to obtain local systems for (some variations of) LL. Multi-modal logics in general have been widely studied in the classical [Bal00] and linear case [DJS93]. However, modular/local systems for multi-modalities were considered only recently [LP15, LP17], and only in the classical case. 
In the first part of this article, we generalize, in a non trivial way, the works op. cit. and present local systems for extensions of multiplicative additive linear logic MALL with simply dependent multimodalities. This (infinite) class of systems includes every extension of MALL with modalities characterized by combinations of the modal axioms $(\mathrm{K}, \mathrm{D}, \mathrm{T}, 4)$ as well as weakening and contraction. This includes, e.g., MALL itself, LL, elementary LL, bounded LL, subexponential LL, as well as very general combinations thereof (item (c) above). Furthermore, using a trivial embedding (by prefixing every subformula with a fitting modality), we can also treat affine LL, relevance logic, and classical logic.

The second part of this article explores how to use LL itself as a meta-level framework for encoding all the systems listed above, which naturally extends the work in [LP15]. Moreover, since such encodings give rise to bipole formulas in LL, they can be easily adapted to other logical frameworks, such as LKF [MV15, MMV16]. Surprisingly enough, these results show that the system SELL [NM09, OPN15] for linear logic with subexponentials can be encoded in linear logic.

We finish the paper by presenting a prover for our systems. The prover is parametric in the axioms, showing how a suitable choice of logical systems can give rise to a general theorem prover. We observe that modularity of the logical framework is of paramount importance for such a generic implementation.

Organization and contributions In Section 2 we consider $L N S_{L L}$, a system with local rules for linear logic using linear nested sequents. The promotion rule of $L N S_{\mathrm{LL}}$ does not require to test the sequent context to be applied, thus making it simpler and more elegant from the theoretical point of view and also more suitable for implementation. We also present a local system for linear logic with bounded exponentials $\left(\mathrm{LNS}_{\mathrm{LL}_{b}}\right.$ ) and $\mathbf{F L N S} \mathrm{LL}_{\mathrm{LL}}$, a focused version of $\mathrm{LNS}_{\mathrm{LL}}$ and $\mathrm{LNS}_{\mathrm{LL}_{b}}$. In Section 3 we extend the concept of simply dependent multimodal logics (SDML) to the linear case. We give a general view of different modalities where MALL is the base logic. We show that different extensions of linear logic such as Elementary Linear Logic and Linear Logic with Subexponentials (SELL) are particular instances of SDML. Section 4 presents linear nested sequents for multimodalities and encodings of SDML into (plain) LL. The results of this section have interesting consequences. We show that SELL, thought to be more expressive than LL, is in fact as expressive as LL. This supports the view that LL is indeed a universal framework that carries itself all the information of its extensions. As a more practical outcome, this result also shows that any implementation of LL could be used to generate a prover for any instance of SDML. However, in this work we decided to implement a general prover parametric to a given SDML. We describe a prototypical tool following this direction. Finally, Section 5 concludes. This paper thus strives at better understanding the role of modalities from a purely syntactic perspective.

\section{Local rules for linear logic}

In this section we propose a system for linear logic with local rules based on the linear nested sequent framework. Although we assume that the reader is familiar with linear logic, we review some of its basic proof theory (see [Tro92] for more details).

\subsection{Linear logic}

Linear logic (LL) is a substructural logic proposed by Girard [Gir87], where not all formulas are allowed to be contracted or weakened. Formulas are built from the following grammar

$$
F::=p\left|p^{\perp}\right| 1|0| \top|\perp| F_{1} \otimes F_{2}\left|F_{1} 8 F_{2}\right| F_{1} \& F_{2}\left|F_{1} \oplus F_{2}\right| \exists x . F|\forall x . F| ? F \mid ! F
$$

and connectives are separated into two classes, the negative: $\perp, \top, \&, \ngtr, \forall, ?$ and the positive: $0,1, \otimes, \oplus, \exists, !$. The polarity of non-atomic formulas is inherited from their outermost connective (e.g., $F \otimes G$ is a positive formula) and any bias can be assigned to atomic formulas [And92]. 


$$
\begin{aligned}
& \overline{p, p^{\perp}} \text { init } \quad \overline{1}^{1} \quad \overline{\Gamma, \top}^{\top} \quad \frac{\Gamma_{1}, F \Gamma_{2}, F^{\perp}}{\Gamma_{1}, \Gamma_{2}} \text { cut } \\
& \frac{\Gamma}{\Gamma, \perp} \perp \quad \frac{\Gamma, F, G}{\Gamma, F \& G} \& \quad \frac{\Gamma, F \quad \Gamma, G}{\Gamma, F \& G} \& \quad \frac{\Gamma, F[y / x]}{\Gamma, \forall x . F} \forall \\
& \frac{\Gamma_{1}, F \quad \Gamma_{2}, G}{\Gamma_{1}, \Gamma_{2}, F \otimes G} \otimes \quad \frac{\Gamma, F_{i}}{\Gamma, F_{1} \oplus F_{2}} \oplus_{i} \quad \frac{\Gamma, F[t / x]}{\Gamma, \exists x . F} \exists \\
& \frac{\Gamma, ? F, ? F}{\Gamma, ? F} \text { cont } \quad \frac{\Gamma}{\Gamma, ? F} \text { weak } \quad \frac{\Gamma, F}{\Gamma, ? F} \text { der } \quad \frac{F, ? \Gamma}{! F, ? \Gamma} \text { prom }
\end{aligned}
$$

Figure 1: Sequent system LL for classical linear logic. In the init rule, $p$ is an atomic formula and in the $\forall$ rule, $y$ is a fresh variable.

LL sequents have the form $\Gamma$ where $\Gamma$ is a multiset of formulae. I.e., we adopt the one sided sequent formulation of classical linear logic, although all the results in this paper could be extended to the intuitionistic (and hence two sided) case. We write $A^{\perp}$ for the negation of the formula $A$, understood as usual by pushing the negation to the atoms using the known dualities, e.g., $(A \otimes B)^{\perp} \equiv A^{\perp} 8 B^{\perp}$. The sequent system $L L$ is presented in Fig. 1. We recall that contraction and weakening of formulas are controlled using the connectives! and ? (called exponentials) and rules cont, weak. The calculus for multiplicative additive linear logic MALL is obtained by removing the modal rules cont, weak, der, prom.

The following formulas are of special interest, since they have classical behavior [GMM98, extended version of Def. 3.1].

Definition 1. A formula $A$ is essentially exponential (Exp) if it is built from the grammar

$$
A:=? B|\perp| A \& A|A \ngtr A| \forall x . A
$$

where $B$ is any linear logic formula.

Proposition 2.1. If $A \in \mathrm{Exp}$, then $A \equiv \mathrm{LL}$ ?A.

The proof is standard, by structural induction and using the fact that formulas in this fragment have negative outermost connective. The result is important since it shows that the context restriction on the promotion rule prom could be softened: instead of only question marked formulas, one could ask for Exp formulas in the context. We observe that the unit $\mathrm{T}$ also satisfies Proposition 2.1, but it has a non-local, positive hidden behavior which we shall discuss in Section 2.3.

\subsection{A linear nested sequent system for linear logic}

In [Str02, GMM98], systems of local rules for linear logic were proposed. While in [Str02] locality was achieved by the use of deep inference [Gug07], in [GMM98] the so called 2-sequents systems were used. In this work we shall study systems with local rules for (possibly multi-) modal systems based on multiplicative-additive linear logic (MALL). For that, we will consider the framework of linear nested sequents (LNS, see [Lel15]), essentially a reformulation of the 2-sequent framework. While in the monomodal case linear nested sequents are simply the 2-sequents of [GMM98] in a different notation, the fact that the nesting is given explicitly means they are much easier adapted to the multimodal setting.

Definition 2. The set LNS of linear nested sequents is given recursively by:

$$
\text { 1. if } \Gamma \text { is a sequent then } \Gamma \in \mathrm{LNS}
$$




$$
\begin{aligned}
& \overline{\mathcal{E} / / p, p^{\perp}} \text { init } \quad \overline{\mathcal{E} / / 1} 1 \quad \frac{\mathcal{E} / / \Gamma, \top}{\top} \quad \frac{\mathcal{G} / / \Gamma_{1}, F \quad \mathcal{G} / / \Gamma_{2}, F^{\perp}}{\mathcal{G} / / \Gamma_{1}, \Gamma_{2}} \text { cut } \\
& \frac{\mathcal{S}\{\Gamma\}}{\mathcal{S}\{\Gamma, \perp\}} \perp \frac{\mathcal{S}\{\Gamma, F, G\}}{\mathcal{S}\{\Gamma, F \& G\}} \ngtr \quad \frac{\mathcal{S}\{\Gamma, F\} \quad \mathcal{S}\{\Gamma, G\}}{\mathcal{S}\{\Gamma, F \& G\}} \& \frac{\mathcal{G} / / \Gamma, F[y / x]}{\mathcal{G} / / \Gamma, \forall x . F} \forall \\
& \frac{\mathcal{G} / / \Gamma_{1}, F \quad \mathcal{G} / / \Gamma_{2}, G}{\mathcal{G} / / \Gamma_{1}, \Gamma_{2}, F \otimes G} \otimes \quad \frac{\mathcal{G} / / \Gamma, F_{i}}{\mathcal{G} / / \Gamma, F_{1} \oplus F_{2}} \oplus_{i} \quad \frac{\mathcal{G} / / \Gamma, F[t / x]}{\mathcal{G} / / \Gamma, \exists F} \exists \\
& \frac{\mathcal{S}\{\Gamma, ? F, ? F\}}{\mathcal{S}\{\Gamma, ? F\}} \text { cont } \quad \frac{\mathcal{S}\{\Gamma\}}{\mathcal{S}\{\Gamma, ? F\}} \text { weak } \quad \frac{\mathcal{S}\{\Gamma, F\}}{\mathcal{S}\{\Gamma, ? F\}} \text { der } \\
& \frac{\mathcal{S}\{\Gamma / / \Delta, ? F\}}{\mathcal{S}\{\Gamma, ? F / / \Delta\}} ? \quad \frac{\mathcal{G} / / \Gamma / / F}{\mathcal{G} / / \Gamma, ! F} !
\end{aligned}
$$

Figure 2: System $\mathrm{LNS}_{\mathrm{LL}}$ for linear logic. In the init rule, $p$ is atomic and in the $\forall$ rule, $y$ is a fresh variable.

\section{2. if $\Gamma$ is a sequent and $\mathcal{G} \in \mathrm{LNS}$ then $\Gamma / / \mathcal{G} \in \mathrm{LNS}$.}

We write $\mathcal{S}\{\Gamma\}$ for denoting a context $\mathcal{G} / / \Gamma / / \mathcal{H}$ where each of $\mathcal{G}$ and $\mathcal{H}$ is either empty or a linear nested sequent. We call each sequent in a linear nested sequent a component and we will denote by $\mathcal{E}$ the empty list and any linear nested sequent $\cdot \| / . / / \cdot$ containing only empty components, also called an empty history. Finally, we slightly abuse notation and abbreviate "linear nested sequent" to LNS.

In Figure 2 we present the system $L N S_{L L}$ with local rules for linear logic. We will call $L N S_{M A L L}$ the system $L N S_{L L}$ restricted to MALL connectives (i.e, without ! and ?).

Observe that the promotion rule has been decomposed into the two rules, ! and ?, both of which are completely local, in the sense that one does not need to check the formula context in order to apply them. More precisely, applying the ! rule enables the creation of the future history, in which the banged formula should be proved. The intended interpretation of a LNS is (writing $8 \Gamma$ for $A_{1} 8 \ldots .8 A_{n}$ if $\Gamma=A_{1}, \ldots, A_{n}$ ):

$$
\begin{array}{lll}
\iota(\Gamma) & :=8 \Gamma \\
\iota(\Gamma / / \mathcal{H}) & :=8 \Gamma 8 ! \iota(\mathcal{H})
\end{array}
$$

Note that, as in [GMM98], the (non invertible) rules for positive connectives can be applied only in the last component (observe that, in LNS, the T is positive, see Section 2.3). This is crucial in order to assure correctness. In fact, a naive linear nested system (where MALL rules could be applied anywhere) would render provable, e.g., the sequent $? A \oplus ? B, !\left(A^{\perp} \& B^{\perp}\right)$, which is not provable in LL. A different possibility for guaranteeing correctness would be to restrict the use of additive connectives. However, the resulting system would be neither local nor modular.

Note however that, unlike in the 2-sequent system of [GMM98], the backwards history is always shared, even in the tensor rule, a fact which is crucial for the encodings of Section 4.2. This is possible due to the fact that only formulas in Exp can jump to higher (already existent) components. More precisely, by induction on the depth of the derivation we obtain (compare [GMM98, Lem. 3.3]):

Proposition 2.2. If $\mathcal{G} / / \Gamma$ is provable in $\mathrm{LNS}_{\mathrm{LL}}$, then $A \in \operatorname{Exp}$ for all formulas $A$ appearing in $\mathcal{G}$.

Theorem 2.1. The linear nested sequent system $\mathrm{LNS}_{\mathrm{LL}}$ is correct and complete w.r.t. $\mathrm{LL}$.

Proof. Observe that, by a permutation-of-rules argument, all the rules in $\mathrm{LNS}_{\mathrm{LL}}$ can be applied in the last component, with the exception of ?. Suppose that $\mathcal{G} / / \Gamma$ is provable in $\mathrm{LNS}_{\mathrm{LL}}$. From Proposition 2.2, every $A \in \mathcal{G}$ can be eagerly decomposed in the rightmost component until either the unit $\perp$ occurs (and the 
formula disappears) or a question-marked formula is reached. That is, the application of the rule ! can be restricted to the case where the context is classical, and this emulates the behavior of the promotion rule.

For the other direction, we simulate a $L L$ derivation bottom-up by a $L N S_{L L}$ derivation which only manipulates the rightmost components. In particular, a (backwards) application of prom is simulated by:

$$
\frac{? \Gamma, F}{? \Gamma, ! F} \text { prom } \vdots \begin{gathered}
\mathcal{E} / / \cdot / / \Gamma, F \\
\frac{\mathcal{E} / / ? \Gamma / / F}{\mathcal{E} / / ? \Gamma, ! F}
\end{gathered} ?
$$

The proof of the last theorem reveals that, in fact, the application of rules in $L^{N} S_{L L}$ can be restricted to the two rightmost components (also compare [GMM98, p. 740]). This justifies the following definition.

Definition 3. A LNS calculus is end-active if in all its rules the rightmost components of the premises are active and the only active components (in premises and conclusion) are the two rightmost ones. The end-active variant of a LNS calculus is the calculus obtained by restricting all rules to be end-active.

Corollary 2.1. The end-active variant of $\mathrm{LNS} \mathrm{L}_{\mathrm{L}}$ is correct and complete w.r.t. $\mathrm{LL}$.

The result above is important for, at least, four reasons:

1. as usual in nested systems, locality comes with a price: the number of possible proofs, hence the proof search space, increases exponentially; with an end-active version of $L N S_{L L}$, the complexity of proof search can be reduced to essentially that of sequent calculus (compare [LP15]);

2. it is possible to define the concept of partially processed rules, opening the possibility of modularly representing non-normal modalities and substructural behaviors (see Section 2.3);

3. it is easy to propose a focused, local system for LL (see Section 2.4);

4. being able to always remember only the last two components makes it possible to propose a labelled version of the linear nested system (see Section 4.1).

\subsection{On bounded exponentials and local $T$}

We note that the local rules for LL presented in Figure 2 take for granted contraction for exponentials. This is reflected in the rules $\otimes$ and cut, that copy the backwards history instead of splitting it.

Example 2.1. The sequent $? p^{\perp}, !(p \otimes p)$ is provable in $\mathrm{LNS} \mathrm{LL}$ and one of the possible proofs is

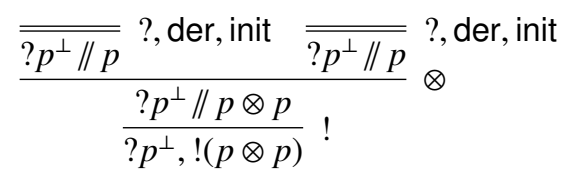

Observe that there is an implicit contraction given by the tensor rule, allowed by Proposition 2.2.

While this is not an issue for LL itself, it becomes problematic, e.g., for linear logic with bounded exponentials $\left(\mathrm{LL}_{b}\right)$, where ? does not allow for contraction nor weakening. In this case, the rules cut and $\otimes$ presented in Figure 2 are not correct. Although it would be possible to simply add the splitting version of such logical rules in order to handle also systems with bounded exponentials, we prefer to utilise the mechanism from [LP15] that can modularly be extended to multi modal logics (Section 3). 


$$
\frac{\mathcal{G}\|\Gamma\| \Delta, ? F}{\mathcal{G}\|\Gamma, ? F\| \Delta} ? \frac{\mathcal{G}\|\Gamma\| F}{\mathcal{G} \| \Gamma, ! F} ， \frac{\mathcal{G} / / \Gamma}{\mathcal{G} \| \Gamma} \mathrm{r} \quad \frac{\mathcal{S}\{\Gamma, F\}}{\mathcal{S}\{\Gamma, ? F\}} \operatorname{der} \quad \frac{\mathcal{G}\|\Gamma\|_{p} \top}{\mathcal{G} \| \Gamma, \top} \operatorname{pt} \frac{\mathcal{G} / / \Gamma \|_{p} \Delta}{\mathcal{G}\|\Gamma, F\|_{p} \Delta} \operatorname{tw} \quad \frac{\mathcal{E} \|_{p} \top}{\top}
$$

Figure 3: The linear nested sequent rules for bounded exponentials $\left(\mathrm{LNS}_{\mathrm{LL}_{b}}\right)$ and partially processed $\mathrm{T}$.

For this, following the idea that the modal LNS rules can be seen as decompositions of standard sequent rules, we introduce the auxiliary nesting operator $\Downarrow$ to capture a state where a sequent rule has been partly processed. In contrast, the intuition for the original nesting // is that the simulation of the application of the modal rule is finished.

Another particularity of linear logic behavior in its LNS version is that the T rule is not invertible. In fact, one should test the emptiness of the backwards history in order to apply the $T$ rule. This is the same behavior of the rule for the unit 1 , with the difference that all formulas in the component where $T$ occurs are weakened. That is, $T$ is, in fact, a composition of two operators: one structural and the other linear. For correctly capturing this behavior locally, we add the nesting operator $\|_{p}$, that first processes the structural step (rule tw), then considers the axiomatic linear behavior of $T$ (rule $T$ ). The definition of linear nested sequents (Def. 2) thus is extended by:

3. if $\Gamma$ and $\Delta$ are sequents, then $\Gamma \rrbracket \Delta \in \mathrm{LNS}$.

4. if $\Gamma$ and $\Delta$ are sequents, then $\Gamma \|_{p} \Delta \in \mathrm{LNS}$.

The interpretation of the new nesting operators is the following:

$$
\begin{aligned}
& \iota(\Gamma \rrbracket \Delta):=8 \Gamma ४ ! 8 \Delta \\
& \iota\left(\Gamma \|_{p} \Delta\right):=8 \Gamma 88 \Delta
\end{aligned}
$$

The system $L N S_{L_{b}}$ has the rules for LNS Observe that, in view of end-active systems, we restrict the occurrence of $\Downarrow$ to the end components. Note also that the sequent in Example 2.1 is not provable in $\mathrm{LNS}_{\mathrm{LL}_{b}}$. In fact, it is straightforward to show correctness and completeness of $\mathrm{LNS}_{\mathrm{LL}_{b}}$ w.r.t. $\mathrm{LL}_{b}$ by noticing that the modal rules in $\mathrm{LNS}_{\mathrm{LL}_{b}}$ only occur in blocks: start with !, apply the ? rule as many times as needed, then finish the block with the release rule $r$ (see Example 2.2). Hence LNS derivations can be translated into standard sequent derivations in $L_{b}$.

Example 2.2. We illustrate the use of the nesting operators $\|$ and $\|_{p}$ in the following derivation, where $p, q$ are atomic formulas.

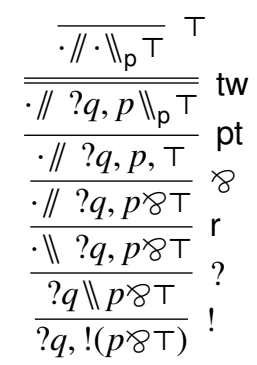

From now on, we will abuse the notation and call $L N S_{L L}$ the end-active LNS system for linear logic with the partial nesting operators $\|$ and $\|_{p}$, where the rules for exponentials and T in Figure 2 are substituted by the respective rules in Figure 3. 
Negative rules

$$
\begin{aligned}
& \frac{\mathcal{G} \| \Uparrow \Psi ; \Gamma}{\mathcal{G} \| \Uparrow \Psi ; \Gamma, \perp} \perp \quad \frac{\mathcal{G} \| \Uparrow \Psi ; \Gamma, F, G}{\mathcal{G} \| \Uparrow \Psi ; \Gamma, F 8 G} 8 \quad \frac{\mathcal{G}\|\Uparrow \Psi ; \Gamma, F \quad \mathcal{G}\| \Uparrow \Psi ; \Gamma, G}{\mathcal{G} \| \Uparrow \Psi ; \Gamma, F \& G} \& \\
& \frac{\mathcal{G} / \Uparrow \Psi ; \Gamma, F[y / x]}{\mathcal{G} \| \Uparrow \Psi ; \Gamma, \forall x . F} \forall \quad \frac{\mathcal{G} \| \Uparrow \Psi, F ; \Gamma}{\mathcal{G} \| \Uparrow \Psi ; \Gamma, ? F} \text { store }
\end{aligned}
$$

Positive rules

$$
\begin{aligned}
& \overline{\mathcal{E} / / \Psi ; \cdot \Downarrow 1} 1 \quad \frac{\mathcal{G} / / \Psi ; \Gamma \|_{p} \Upsilon ; \Delta \Downarrow \top}{\mathcal{E} \|_{p} \Psi ; \cdot \Downarrow T} T \quad \frac{\mathcal{G} / / \Psi ; \Gamma, F \|_{p} \Upsilon ; \Delta \Downarrow \top}{t w} \\
& \frac{\mathcal{G} / / \Psi ; \Gamma_{1} \Downarrow F \quad \mathcal{G} / / \Psi ; \Gamma_{2} \Downarrow G}{\mathcal{G} / / \Psi ; \Gamma_{1}, \Gamma_{2} \Downarrow F \otimes G} \otimes \frac{\mathcal{G} / / \Psi ; \Gamma \Downarrow F_{i}}{\mathcal{G} / / \Psi ; \Gamma \Downarrow F_{1} \oplus F_{2}} \oplus_{i} \\
& \frac{\mathcal{G} / / \Psi ; \Gamma \Downarrow F[t / x]}{\mathcal{G} / / \Psi ; \Gamma \Downarrow \exists x . F} \exists \quad \frac{\mathcal{G} / / \Psi ; \Gamma \| \Upsilon, G ; \Delta \Downarrow F}{\mathcal{G} / / \Psi, G ; \Gamma \| \Upsilon ; \Delta \Downarrow F} ? \quad \frac{\mathcal{G} \| \Uparrow \Psi ; \Gamma \Downarrow \cdot ; \cdot \Downarrow F}{\mathcal{G} / / \Psi ; \Gamma \Downarrow ! F} !
\end{aligned}
$$

Identity and Decide and Release rules

$$
\begin{gathered}
\frac{\mathcal{E} / / \Psi ; A \Downarrow A^{\perp}}{\mathrm{I}_{1}} \quad \overline{\mathcal{E} / / \Psi, A ; \cdot \Downarrow A^{\perp}} \mathrm{I}_{2} \\
\frac{\mathcal{G} / / \Psi, F ; \Gamma \Downarrow F}{\mathcal{G} / \| \Psi, F ; \Gamma} \mathrm{D}_{\mathrm{c}} \quad \frac{\mathcal{G} / / \Psi ; \Gamma \Downarrow P}{\mathcal{G} / \| \Uparrow \Psi ; \Gamma, P} \mathrm{D}_{\mathrm{l}}, P \text { is positive } \quad \frac{\mathcal{G} / / \Psi ; \Gamma \|_{\mathrm{p}} \cdot ; \Downarrow \mathrm{T}}{\mathcal{G} / \| \Uparrow \Psi ; \Gamma, \top} \mathrm{D}_{\mathrm{p}} \\
\frac{\mathcal{G} / / \Uparrow \Psi ; \Gamma, F}{\mathcal{G} \| \Psi ; \Gamma \Downarrow F} \mathrm{R}_{\mathrm{r}} \quad \frac{\mathcal{G} / \| \Uparrow \Psi ; \Gamma, N}{\mathcal{G} / / \Psi ; \Gamma \Downarrow N} \mathrm{R}_{\mathrm{n}}, N \text { is negative }
\end{gathered}
$$

Figure 4: Focused proof search in nested linear logic $\mathbf{F L N S} \mathbf{L L}_{\mathrm{LL}}$ In the Identity rules, $A$ is a negative literal.

\subsection{A focused system for $L N S_{L L}$}

Focusing [And92] is a discipline on proofs aiming at reducing the non-determinism during proof search. Focused proofs can be interpreted as normal form proofs. It is based on the fact that the negative connectives have invertible rules, while positive connectives have non-invertible rules. This separation induces a two phase proof construction: a negative phase, where no backtracking on the selection of inference rules is necessary, and a positive phase, where choices within inference rules can lead to failures for which one may need to backtrack.

We separate the context of sequents in two: the set $\Psi$ will always denote the unbounded context, containing only question-marked formulas, while $\Gamma$ is a general linear context. We will differentiate

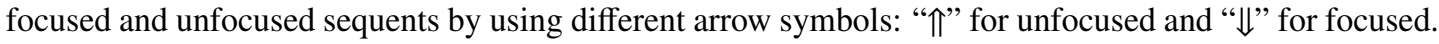
In this way, $\mathbf{F L N S}_{\mathrm{LL}}$ contains two types of sequents in the components:

i. $\Uparrow \Psi ; \Gamma$ is an unfocused sequent.

ii. $\Psi ; \Gamma \Downarrow F$ is a focused sequent.

We call a literal either an atom or a negated atom and we recall that negation is involutive in linear logic, implying that, for any formula $F,\left(F^{\perp}\right)^{\perp} \equiv F$.

The rules for the nested (weak) focused system for $L L$ are depicted in Figure 4. The focusing is weak since one could focus on a positive formula even if the context has negative ones. One could avoid that by either (1) restricting the context $\Gamma$ in the decision rules so to have only positive or atomic formulas; or (2) presenting a synthetic version of the system, where the logical content of the phases of focusing are 


$$
\begin{gathered}
\mathrm{C} ! B \multimap ! B \otimes ! B \quad \mathrm{~W} ! B \multimap 1 \\
\mathrm{~K} !(A \multimap B) \multimap(! A \multimap ! B) \quad 4 ! A \multimap ! ! A \quad \mathrm{D} ! A \multimap ? A \quad \mathrm{~T} ! A \multimap A
\end{gathered}
$$

Figure 5: Structural and modal axioms.

abstracted from the level of formulas to the level of nested sequents (see, e.g., [CMS16]). While (1) goes against the idea of having only local rules, (2) is easily achieved by a simple adaptation of the system presented in [CMS16].

It is worth noting that, unlike focusing in sequent presentations for LL [And92], in the system FLNS $_{\mathrm{LL}}$ the banged formula in the ! rule does not lose focus. This is due to the use of the partial nesting operator $\Downarrow$. Observe, however, that the only action that can be done in this focused step is moving classical formulas between nested contexts. This traduces, in a finer way, the positive/negative nature of ! (resp. the dual negative/positive behavior of ?): while creating a new component is a positive action, moving classical formulas between components is a negative step (resp. classical formulas can be moved only after the creation of components).

Regarding $T$, we still consider it negative, as a linear logic connective. Hence any classical focusing on a $T$ (application of $D_{c}$ ) will be necessarily followed by a release (rule $R_{n}$ ), while the rule $D_{1}$ can never be applied. But, once in the linear context, $T$ can be focused using $D_{p}$, and the proof terminates in a positive phase, due to the linear (positive) behavior of $T$ in $L N S_{L L}$.

\section{Simply dependent multimodal linear logics}

In this section, we extend the concept of simply dependent multimodal logics [Dem00] to the linear case. That is, we study different modalities, having MALL as the base logic. Consider the structural axioms $\mathrm{C}, \mathrm{W}$ and axioms for modalities $\{\mathrm{K}, 4, \mathrm{D}, \mathrm{T}\}$, shown in Figure $5 .{ }^{1}$ We start by recalling the standard observation that, due to the transitive and reflexive behavior of ? in the promotion rule and the dereliction rule, the linear logic modalities have a flavor of the modalities of S4 (Axioms 4 and T).

Hence, on substituting the modal axioms but maintaining MALL as the base logic, one obtains, in a modular way, a class of different logics. The following definition is an adaption of the modular presentation of simply dependent multimodal logics appearing in [Ach16].

Definition 4. A simply dependent multimodal logical system (SDML) is given by a triple $(I, \preccurlyeq, F)$, where I is a set of indices, $(I, \preccurlyeq)$ is a pre-order (i.e., reflexive and transitive), and $F$ is a mapping from I to $2^{\{\mathrm{D}, \mathrm{T}, 4, \mathrm{C}, \mathrm{W}\}}$

Here we assume that all the logics include the distribution axiom $!(A \multimap B) \multimap(! A \multimap ! B)$ (taken as a zero-premiss rule) plus the rule of necessitation $A / ! A$ and we will use (classical) MALL as the base logic.

Definition 5. If $(I \leqslant, F)$ is a SDML, then the logic described by $(I, \leqslant, F)$ has modalities $!^{i}$, ? for every $i \in I$, with the rules of MALL (including cut) of Fig. 1, together with rules and axioms for the modality $i$ given by the necessitation rule and the $\mathrm{K}$ axiom for $!^{i}$ as well as the axioms $F(i)$, and interaction axioms $! !^{j} A \multimap !^{i} A$ for every $i, j \in I$ with $i \leqslant j$, understood as zero-premiss rules.

Several known logical systems can be seen as particular instances of this definition:

Example 3.1. LL can be seen as a trivial case of SDML, where $I=\{i\}$ and $F(i)=\{4, \mathrm{~T}, \mathrm{C}, \mathrm{W}\}$.

\footnotetext{
${ }^{1}$ To increase readability, we represent $A^{\perp} \ngtr B$ by $A \multimap B$.
} 
Example 3.2. Another trivial case of SDML is Elementary Linear Logic ELL [Gir98], with index set I also a singleton and $F(i)=\{\mathrm{D}, \mathrm{C}, \mathrm{W}\}$ [GMM98].

Example 3.3 (Structural variants of MALL). Adding combinations of contraction cont and/or weakening weak for arbitrary formulae to (cut-free) MALL yields, respectively, classical logic CL $=$ MALL + \{cont, weak\}, affine linear logic aLL $=\mathrm{MALL}+$ weak and relevant logic $\mathrm{R}=\mathrm{MALL}+$ cont. In order to embed the logics above into $\mathrm{LL}$, let $\alpha \in\{\mathrm{CL}, \mathrm{aLL}, \mathrm{R}\}$ and consider a pair $?^{\alpha}, !^{\alpha}$ of modalities with $F(\alpha)=\{\mathrm{T}, 4\} \cup \mathcal{A}$ where $\mathcal{A} \subseteq\{\mathrm{C}, \mathrm{W}\}$ is the set of axioms whose corresponding rules are in $\alpha$. The translation $\tau_{\alpha}$ then prefixes every subformula with the modality ? ${ }^{\alpha}$. For $\mathcal{L} \in\{\mathrm{CL}, \mathrm{aLL}, \mathrm{R}\}$ it is then straightforward to show that a sequent $\Gamma$ is cut-free derivable in $\mathcal{L}$ iff its translation $\tau_{\alpha}(\Gamma)$ is cut-free derivable in the logic described by $(\{\alpha\}, \preccurlyeq, F)$ with $\leqslant$ the obvious relation and $F$ as given above.

Lemma 3.1 (Propagation properties). For every logic $\mathcal{L}$ described by a SDML $(I, \preccurlyeq, F)$ and indices $i, j \in I$ with $i \leqslant j$ we have:

1. If $!^{i} F \multimap F \in \mathcal{L}$, then $!^{j} F \multimap F \in \mathcal{L}$, i.e., axiom T propagates upwards along $\leqslant$;

2. If $!^{i} F \multimap ?^{i} F \in \mathcal{L}$, then $!^{j} F \multimap ?^{j} F \in \mathcal{L}$, i.e., axiom $\mathrm{D}$ propagates upwards along $\preccurlyeq$;

3. If $!^{i} F \multimap 1 \in \mathcal{L}$, then $!^{j} F \multimap 1 \in \mathcal{L}$, i.e., weakening propagates upwards along $\preccurlyeq$.

Proof. Using the axioms and the fact that if $i \leqslant j$, then the logic contains the interaction axiom $!^{j} F \multimap !^{i} F$ (and hence also also $?^{i} F \multimap ?^{j} F$ ). In particular, for (1) we have $!^{j} F \multimap !^{i} F$ and $!^{i} F \multimap F$, hence also $!^{i} F \multimap F$. For (2) we have $!^{j} F \multimap !^{i} F$ which together with $!^{i} F \multimap ? i F$ and $?^{i} F \multimap ?^{j} F$ gives $!^{j} F \multimap ?^{j} F$. Similarly for (3).

Hence w.l.o.g. we may assume that every SDML is upwardly closed with respect to the axioms T, D and W. To obtain cut-free calculi we need to stipulate that the simply dependent multimodal systems are upwardly closed with respect to the axioms 4 and $\mathrm{C}$ as well.

Definition 6. A SDML $(I, \leqslant, F)$ is suitable if it is upwardly closed with respect to axioms 4 and $\mathrm{C}$, i.e., if for every $i, j \in I$ with $i \leqslant j$ it satisfies:

1. if $4 \in F(i)$, then $4 \in F(j)$

2. if $\mathrm{C} \in F(i)$, then $\mathrm{C} \in F(j)$.

Using the methods of [LP13, Lel13] adapted to the substructural context we obtain correct and cut-free complete (standard) sequent systems for suitable SDML as follows.

Definition 7. Given a suitable SDML $(I, \leqslant, F)$, and writing $\uparrow(i)$ for $\{j \in I: i \leqslant j\}$ and $\uparrow^{4}(i)$ for $\{j \in I: i \leqslant j$ and $4 \in F(j)\}$, the sequent calculus $\mathrm{G}_{(I, \ll, F)}$ :

- contains the MALL rules without cut;

- contains contraction (resp. weakening) rules for every ? with $\mathrm{C} \in F(i)$ (resp. $\mathrm{W} \in F(i)$ );

- contains the rules in Figure 6.

We prove cut elimination for the systems $\mathrm{G}_{(I, \leqslant, F)}$ using an auxiliary lemma formulated only for modalised formulas. If $(I, \leqslant, F)$ is a suitable SDML and $\mathcal{D}$ is a derivation using the rules of $\mathrm{G}_{(I, \leqslant, F)}$ as well as cut, we write $\mathrm{rk}_{\text {cut }}(\mathcal{D})$ for the cutrank of $\mathcal{D}$, i.e., the maximal complexity of cutformulas occurring in $\mathcal{D}$. We also write $A^{n}$ for the multiset $A, \ldots, A$ containing $n$ copies of $A$, and similarly for $\Gamma^{n}$. 
Lemma 3.2. If $(I, \preccurlyeq, F)$ is a suitable $\mathrm{SDML}$ and for $i \in I$ there are $\mathrm{G}_{(I, \leqslant)}$-derivations $\mathcal{D}_{1}, \mathcal{D}_{2}$ of $\Gamma_{1}, !^{i} F$ and $\Gamma_{2},\left(\left(!^{i} F\right)^{\perp}\right)^{n}$ respectively with $\mathrm{rk}_{\text {cut }}\left(\mathcal{D}_{1}\right) \leq|F| \geq \mathrm{rk}_{\text {cut }}\left(\mathcal{D}_{2}\right)$ and $n>1$ only if $\mathrm{C} \in F(i)$, then there is $a \mathrm{G}_{(I, \ll, F)}$-derivation $\mathcal{D}$ of $\Gamma_{1}, \Gamma_{2}$ with $\mathrm{rk}_{\text {cut }}(\mathcal{D}) \leq|F|$.

Proof. By induction on the sum of the depths $d_{1}$ and $d_{2}$ of the derivations $\mathcal{D}_{1}$ and $\mathcal{D}_{2}$ respectively.

If one of $d_{1}, d_{2}$ is 0 , then the conclusion of the corresponding derivation must be the conclusion of the $T$ rule. But then the sequent $\Gamma_{1}, \Gamma_{2}$ also is derived using the $T$ rule.

If $d_{1}+d_{2}=k+1$ and the formula $!^{i} F$ is not principal in the last applied rule in $\mathcal{D}_{1}$, as usual we apply the induction hypothesis on the premiss(es) of that rule followed by the same rule. If the formula $!^{i}$ is principal in the last applied rule in $\mathcal{D}_{1}$ and the last applied rule in $\mathcal{D}_{2}$ is not a modal rule, contraction or weakening on the formula $\left(!^{i} F\right)^{\perp}=?^{i} F^{\perp}$, we apply the induction hypothesis on the premiss(es) of that rule, followed by an application of the same rule and possibly applications of cont. E.g., if the last applied rule was $\otimes$ the derivation $\mathcal{D}_{2}$ ends in

$$
\frac{\left(?^{i} F^{\perp}\right)^{n_{1}}, \Sigma_{1}, G \quad\left(?^{i} F^{\perp}\right)^{n_{2}}, \Sigma_{2}, H}{\left(?^{i} F^{\perp}\right)^{n_{1}+n_{2}} \Sigma_{1}, \Sigma_{2}, G \otimes H} \otimes
$$

for some $n_{1}, n_{2}$ with $n_{1}+n_{2}=n$. Note that by assumption of the lemma, $n_{1}+n_{2}>1$ only if $\mathrm{C} \in F(i)$. Thus by induction hypothesis we obtain derivations $\mathcal{D}_{1}^{\prime}$ and $\mathcal{D}_{2}^{\prime}$ with cutrank at most $|F|$ of the sequents

$$
\Gamma_{1}, \Sigma_{1}, G \quad \text { and } \quad \Gamma_{1}, \Sigma_{2}, H
$$

and an application of the rule $\otimes$ yields

$$
\Gamma_{1}, \Gamma_{1}, \Sigma_{1}, \Sigma_{2}, G \otimes H
$$

However, since the formula $!^{i} F$ was principal in the last applied rule of $\mathcal{D}_{1}$, that rule must have been the rule $!^{i}$, and hence $\Gamma_{1}$ has the form $?^{j_{1}} \Delta_{j_{1}}, \ldots, ?^{j_{\ell}} \Delta_{j_{\ell}}$ for indices $j_{1}, \ldots, j_{\ell} \in \uparrow(i)=\{t \in I: i \leqslant t\}$. Moreover, since $\mathrm{C} \in F(i)$ and the $\operatorname{SDML}(I, \leqslant, F)$ is suitable, this means that $\mathrm{C} \in F(t)$ for $t=j_{1}, \ldots, j_{\ell}$ as well. Hence we can now apply cont to obtain the desired sequent $\Gamma_{1}, \Sigma_{1}, \Sigma_{2}, G \otimes H$.

If the formula $\left(!^{i} F\right)^{\perp}$ was principal in the last applied rule of $\mathcal{D}_{1}$ and the last applied rule in $\mathcal{D}_{2}$ is cont on the formula $\left(!^{i} F\right)^{\perp}$, then we simply apply the induction hypothesis on the premiss of that rule.

If the formula $\left(!^{i} F\right)^{\perp}$ was principal in the last applied rule of $\mathcal{D}_{1}$ and the last applied rule in $\mathcal{D}_{2}$ is weak then by Lemma 3.1 we have $\mathrm{W} \in F(t)$ for every $t$ with $i \leqslant t$. Hence, using the fact that since the last applied rule in $\mathcal{D}_{1}$ is a modal rule, $\Gamma_{1}$ consists only of formulae of the form ? $H$ for $t$ with $i \leqslant t$, we may simply apply weak several times to obtain $\Gamma_{1}, \Gamma_{2}$.

If the formula $\left(!^{i} F\right)^{\perp}$ was principal in the last applied rule of $\mathcal{D}_{1}$ and $\mathcal{D}_{2}$ ends in a modal rule we first apply the induction hypothesis on the premiss of that rule to eliminate the occurrences of $\left(!^{i} F\right)^{\perp}$ in the context, i.e., those also occurring in the premiss. Then we eliminate the remaining occurrences of $F^{\perp}$ using standard cuts with rank $|F|$. Finally, we contract the superfluous occurrences of $\Gamma_{1}$, again using that if cont $\in F(i)$, then cont $\in F(j)$ for all $j$ with $i \leqslant j$. E.g., if the last applied rule in $\mathcal{D}_{2}$ was $!^{j}$ for some $j$ with $j \leqslant i$, then that derivation ends in

$$
\frac{\left(F^{\perp}\right)^{n_{1}}, \Delta_{j_{1}}, \ldots, \Delta_{j_{k}}, ?^{i}\left(F^{\perp}\right)^{n_{2}}, ?^{\ell_{1}} \Pi_{\ell_{1}}, \ldots, ?^{\ell_{m}} \prod_{\ell_{m}}, G}{\left(?^{i} F^{\perp}\right)^{n_{1}}, ?^{j_{1}} \Delta_{j_{1}}, \ldots, ?^{j_{k}} \Delta_{j_{k}}, ?^{i}\left(F^{\perp}\right)^{n_{2}}, ?^{\ell_{1}} \prod_{\ell_{1}}, \ldots, ?^{\ell_{m}} \prod_{\ell_{m}}, !^{j} G} !^{j}
$$

Applying the induction hypothesis on the premiss of this rule yields a derivation of the sequent

$$
\left(F^{\perp}\right)^{n_{1}}, \Delta_{j_{1}}, \ldots, \Delta_{j_{k}}, \Gamma_{1}, ?^{\ell_{1}} \Pi_{\ell_{1}}, \ldots, ?^{\ell_{m}} \Pi_{\ell_{m}}, G
$$




$$
\begin{aligned}
& \frac{\Gamma_{j_{1}}, \ldots, \Gamma_{j_{k}}, ?^{\ell_{1}} \sum_{\ell_{1}}, \ldots, ?^{\ell_{m}} \sum_{\ell_{m}}, A}{?^{j_{1}} \Gamma_{j_{1}}, \ldots, ?^{j_{k}} \Gamma_{j_{k}}, ?^{\ell_{1}} \Sigma_{\ell_{1}}, \ldots, ?^{\ell_{m}} \sum_{\ell_{m}},{ }^{i} A} \mathrm{~K} 4_{i} \\
& \frac{\Gamma_{j_{1}}, \ldots, \Gamma_{j_{k}}, ?^{\ell_{1}} \Sigma_{\ell_{1}}, \ldots, ?^{\ell_{m}} \Sigma_{\ell_{m}}}{?^{j_{1}} \Gamma_{j_{1}}, \ldots, ?^{j_{k}} \Gamma_{j_{k}}, ?^{\ell_{1}} \Sigma_{\ell_{1}}, \ldots, ?^{\ell_{m}} \Sigma_{\ell_{m}}} \mathrm{D}_{i}(\text { for } \mathrm{D} \in F(i)) \quad \frac{\Gamma, \Gamma_{j_{1}}, \ldots, \Gamma_{j_{k}}}{\Gamma, ?^{j_{1}} \Gamma_{j_{1}}, \ldots, ?^{j_{k}} \Gamma_{j_{k}}} \mathrm{~T}_{i}(\text { for } \mathrm{T} \in F(i))
\end{aligned}
$$

Figure 6: System $\mathrm{G}_{(I, \leqslant, F)}$ for modal sequent rules for suitable SDML, where $j_{1}, \ldots, j_{k} \in \uparrow(i)$ and $\ell_{1}, \ldots, \ell_{m} \in \uparrow^{4}(i)$ in each of the rules.

Since $\Gamma_{1}, !^{i} F$ is the conclusion of the rule $!^{i}$, the derivation $\mathcal{D}_{1}$ ends in

$$
\frac{\Sigma_{q_{1}}, \ldots, \Sigma_{q_{u}}, ?^{r_{1}} \Omega_{r_{1}}, \ldots, ?^{r_{v}} \Omega_{r_{v}}, F}{?^{q_{1}} \Sigma_{q_{1}}, \ldots, ?^{q_{u}} \Sigma_{q_{u}}, ?^{r_{1}} \Omega_{r_{1}}, \ldots, ?^{r_{v}} \Omega_{r_{v}}, !^{i} F} !^{i}
$$

for some $q_{1}, \ldots, q_{u}, r_{1}, \ldots, r_{v} \in \uparrow(i)$ with $4 \in F\left(r_{s}\right)$ for $s=1, \ldots, v$. Hence, writing $\Xi$ for the multiset $\Sigma_{q_{1}}, \ldots, \Sigma_{q_{u}}, ?^{r_{1}} \Omega_{r_{1}}, \ldots, ?^{r_{v}} \Omega_{r_{v}}$, by $n_{1}$ applications of cut we obtain the sequent

$$
\Xi^{n_{1}}, \Delta_{j_{1}}, \ldots, \Delta_{j_{k}}, \Gamma_{1}, ?^{\ell_{1}} \prod_{\ell_{1}}, \ldots, ?^{\ell_{m}} \prod_{\ell_{m}}, G
$$

By transitivity of $\leqslant$ and $j \leqslant i$ we have that $\uparrow(i) \subseteq \uparrow(j)$, and moreover, since $(I, \leqslant, F)$ is suitable, we know that if $n_{2} \neq 0$ and hence $4 \in F(i)$, then also $4 \in F(t)$ for every $t$ with $i \leqslant t$. Thus we can apply the rule $!^{j}$ to the above sequent to obtain

$$
\Gamma_{1}^{n_{1}}, ?^{j_{1}} \Delta_{j_{1}}, \ldots, ?^{j_{k}} \Delta_{j_{k}}, \Gamma_{1}, ?^{\ell_{1}} \Pi_{\ell_{1}}, \ldots, ?^{\ell_{m}} \Pi_{\ell_{m}}, !^{j} G
$$

Finally, if $n>1$ then by assumption we have $\mathrm{C} \in F(i)$ and thus also $\mathrm{C} \in F(t)$ for every $t$ with $i \leqslant t$. Hence we can apply cont to the formulae in $\Gamma_{1}$ to obtain the desired

$$
\Gamma_{1}, ?^{j_{1}} \Delta_{j_{1}}, \ldots, ?^{j_{k}} \Delta_{j_{k}}, ?^{\ell_{1}} \prod_{\ell_{1}}, \ldots, ?^{\ell_{m}} \prod_{\ell_{m}}, !^{j} G
$$

If either of $n_{1}, n_{2}$ is 0 , the case is adapted in the obvious way. The cases where the last applied rule in $\mathcal{D}_{2}$ was $\mathrm{D}_{j}$ or $\mathrm{T}_{j}$ are similar.

Theorem 3.1. Let $(I, \preccurlyeq, F)$ be a suitable SDML. Then the rule cut is admissible in $\mathrm{G}_{(I, \leqslant, F)}$, i.e., if the sequents $\Gamma_{1}, F$ and $\Gamma_{2}, F^{\perp}$ are derivable in $\mathrm{G}_{(I, \ll, F)}$, then so is the sequent $\Gamma_{1}, \Gamma_{2}$.

Proof. As usual by induction on the tuples $\left\langle|F|, d_{1}+d_{2}\right\rangle$ in the lexicographic ordering, where $|F|$ is the complexity of $F$ and $d_{1}$ and $d_{2}$ are the depths of the derivations of the sequents $\Gamma_{1}, F$ and $\Gamma_{2}, F^{\perp}$ respectively. The cases where the main connective of $F$ is propositional or a quantifier are dealt with as usual. If $F$ is of the form $!^{i} G$ we appeal to Lemma 3.2 and the induction hypothesis.

As usual, the admissibility of the cut rule in the sequent system implies completeness w.r.t. the axiomatic description of the logic.

Theorem 3.2. Given a suitable $\operatorname{SDML}(I, \leqslant, F)$, the sequent system $\mathrm{G}_{(I, \leqslant, F)}$ is correct and cut-free complete for the logic described by $(I, \leqslant, F)$.

Proof. Since we take every modality $!^{i}$ to be an extension of K, i.e., satisfying the distribution axiom $!^{i}(A \multimap B) \multimap\left(!^{i} A \multimap !^{i} B\right)$ and the rule of necessitation $A / !^{i} A$, we assume the standard K-rules

$$
\frac{\Gamma, A}{?^{i} \Gamma, !^{i} A}
$$

for every index $i$. In presence of these rules we have: 
- The rule

$$
\frac{\Gamma_{j_{1}}, \ldots, \Gamma_{j_{k}}, ?^{\ell_{1}} \Sigma_{\ell_{1}}, \ldots, ?^{\ell_{m}} \sum_{\ell_{m}}, A}{?^{j_{1}} \Gamma_{j_{1}}, \ldots, ?^{j_{k}} \Gamma_{j_{k}}, ?^{\ell_{1}} \Sigma_{\ell_{1}}, \ldots, ?^{\ell_{m}} \sum_{\ell_{m}}, !^{i} A} \mathrm{~K} 4_{i}
$$

where $j_{1}, \ldots, j_{k} \in \uparrow(i)$ and $\ell_{1}, \ldots, \ell_{m} \in \uparrow^{4}(i)$ is equivalent (in the system with cut and the interaction axioms) to the axiom

$$
\bigotimes_{s=1}^{k} \bigotimes !^{j_{s}} \Gamma_{j_{s}} \otimes \bigotimes_{t=1}^{m} \bigotimes !^{\ell_{t}} \Sigma_{\ell_{t}} \multimap !^{i}\left(\bigotimes_{s=1}^{k} \bigotimes \Gamma_{j_{s}} \otimes \bigotimes_{t=1}^{m} \bigotimes !^{\ell_{t}} \Sigma_{\ell_{t}}\right)
$$

This is seen by inserting $\bigotimes_{s=1}^{k} \otimes \Gamma_{j_{s}} \otimes \bigotimes_{t=1}^{m} \otimes !^{\ell_{t}} \Sigma_{\ell_{t}}$ for the formula $A$ in the rule, deriving the axiom (1) on the one hand, and using the K-rule for $!^{i}$ followed by a number of cuts with the interaction axioms and the axioms $!^{j} A \multimap !^{j} !^{j} A$ for $j \in \uparrow^{4}(i)$ on the other hand. Axiom (1) is seen to be valid using the derivable axiom $\left(!^{i} B_{1} \otimes \cdots \otimes !^{i} B_{n}\right) \multimap !^{i}\left(B_{1} \otimes \cdots \otimes B_{n}\right)$, the interaction axioms and the axioms $!^{j} A \multimap !^{j} !^{j} A$ for $j \in \uparrow^{4}(i)$. This proves correctness of the rule $\mathrm{K} 4_{i}$.

- Similarly, rule $\mathrm{D}_{i}$ is seen to be equivalent (using cut, interaction- and 4-axioms) to the axiom

$$
\left(\bigotimes_{s=1}^{k} !^{i} \bigotimes \Gamma_{j_{s}} \otimes \bigotimes_{t=1}^{m} !^{i} \bigotimes !^{\ell_{t}} \Sigma_{\ell_{t}}\right) \multimap ?^{i}\left(\bigotimes_{s=1}^{k} \bigotimes \Gamma_{j_{s}} \otimes \bigotimes_{t=1}^{m} \bigotimes !^{t_{t}} \Sigma_{\ell_{t}}\right)
$$

whose validity follows from validity of the formula $!^{i} B_{1} \otimes \cdots \otimes !^{i} B_{n} \multimap ?^{i}\left(B_{1} \otimes \cdots \otimes B_{n}\right)$ for logics with $\mathrm{D} \in F(i)$, showing correctness of the rule $\mathrm{D}_{i}$.

- Finally, rule $T_{i}$ is derivable by using cuts and the fact that the T axiom for modality $i$ is equivalent to provability of the sequent $A^{\perp}, ?^{i} A$.

Hence the system $\mathrm{G}_{(I, \leqslant, F)}$ is correct.

For completeness it is straightforward to derive the interaction axioms and the axioms for each logic using the sequent rules. The result then follows from cut elimination of system $\mathrm{G}_{(I, \leqslant, F)}$ (Thm. 3.1) and from the fact that weakening, contraction, and the axiom 4 are upwardly closed.

\subsection{Linear logic with subexponentials}

As one of the main examples of logics given by a suitable SDML, we consider linear logic with subexponentials (SELL - see [DJS93, NM09, OPN15]). SELL shares with LL all its connectives except the exponentials: instead of having a single pair of exponentials ! and ?, SELL may contain as many subexponentials, written $!^{a}$ and $?^{a}$, as one needs.

The proof system for SELL is specified by a subexponential signature $\Sigma=\langle I, \leq, U\rangle$, where $I$ is a set of labels, $U \subseteq I$ is a set specifying which subexponentials allow weakening and contraction, $\leq$ is a pre-order among the elements of $I$ that is upwardly closed with respect to $U$, i.e., if $a \in U$ and $a \leq b$, then $b \in U$.

The system SELL is constructed by adding the following rules to MALL:

$$
\frac{?^{a_{1}} F_{1}, \ldots ?^{a_{n}} F_{n}, G}{?^{a_{1}} F_{1}, \ldots ?^{a_{n}} F_{n}, !^{a} G} !^{a} \quad \frac{\Gamma, G}{\Gamma, ?^{a} G} ?^{a}
$$

where the rule $!^{a}$ has the side condition that $a \leq a_{i}$ for all $i$. Moreover, for all indices $a \in U$, we add the usual rules for weakening and contraction. 


$$
\begin{aligned}
& \frac{\mathcal{G}\left\|^{k} \Gamma\right\|^{i} \Delta, F}{\mathcal{G}\left\|^{k} \Gamma, ?^{j} F\right\|^{i} \Delta} ?^{i}{ }_{\mathrm{k}}(\text { for } j \in \uparrow(i)) \quad \frac{\mathcal{G}\left\|^{k} \Gamma\right\|^{i} F}{\mathcal{G} \|^{k} \Gamma, !^{i} F} !^{i} \quad \frac{\mathcal{G} \|^{i} \Gamma}{\mathcal{G} \|^{i} \Gamma} \mathrm{r} \\
& \left.\frac{\mathcal{G}\left\|^{k} \Gamma\right\|{ }^{i} \Delta, ?^{j} F}{\mathcal{G}\left\|^{k} \Gamma, ?^{j} F\right\|^{i} \Delta} ?^{i}{ }_{4}\left(\text { for } j \in \uparrow^{4}(i)\right) \quad \frac{\mathcal{G}\left\|^{k} \Gamma\right\|^{i} F}{\mathcal{G} \|^{k} \Gamma, ?^{i} F} ?^{i}{ }_{\mathrm{d}}(\text { for } \mathrm{D} \in F(i)) \quad \frac{\mathcal{G} \|^{k} \Gamma, F}{\mathcal{G} \|^{k} \Gamma, ?^{i} F} ?_{\mathrm{t}}^{i} \text { (for } \mathrm{T} \in F(i)\right)
\end{aligned}
$$

Figure 7: The linear nested rules for the exponentials in $L N S_{(I, \leqslant, F)}$.

Depending on the pre-order, proofs in SELL can be interpreted as concurrent processes with different behaviors. For example, formulas having the shape $?^{a} F$ may represent processes occurring inside the space/location $a$, while $?^{a} !^{a} F$ confines the process $F$ to that space. In a series of works [NOP13, PON14, OPN15], SELL was used in order to capture modal behaviors in concurrent systems such as time, knowledge, probability, fuzziness, costs and preferences.

Going through the definitions, it is straightforward to check that SELL can be seen as an instance of our framework.

Example 3.4. SELL with signature $\langle I, \preccurlyeq, U\rangle$, is $\mathrm{G}_{(I, \leqslant, F)}$ for a suitable $\operatorname{SDML}$ determined by $(I, \preccurlyeq, F)$ where $F(i)=\{\mathrm{T}, 4, \mathrm{C}, \mathrm{W}\}$ if $i \in U$ and $F(i)=\{\mathrm{T}, 4\}$ otherwise.

\section{Linear nested sequents for multimodalities}

Following the ideas of Section 2, we introduce now local calculi for logics given by suitable SDMLs. However, in order to convert $\mathrm{G}_{(I, \leqslant, F)}$ sequent systems into LNS systems, we need to modify the linear nested setting to account for all the different non-invertible rules. For this, given a SDML $(I, \preccurlyeq, F)$ we introduce nesting operators $/ /^{i}$ and their unfinished versions $\|^{i}$ for every $i \in I$ as in Sec. 2.3, and change the interpretation so that they are interpreted by the corresponding modality:

$$
\begin{aligned}
\iota(\Gamma):=\varnothing \Gamma \\
\iota\left(\Gamma /\left.\right|^{i} \mathcal{H}\right):=\iota\left(\Gamma \|^{i} \mathcal{H}\right):=\varnothing \Gamma 8 !^{i} \iota(\mathcal{H})
\end{aligned}
$$

Again, the operators $\|^{i}$ are used to handle bounded exponentials (see Section 2.3), and indicate that the standard sequent rule for the modality indexed by $i$ has been partially processed.

If $(I, \preccurlyeq, F)$ is a SDML, the linear nested system $\operatorname{LNS}_{(I, \leqslant, F)}$ is given by the rules for LNS $\mathrm{SALL}_{\text {with the }}$ rules for $\top$ from Figure 3 plus the rules in Figure 7, together with weakening/contraction LNS rules for every ${ }^{i}$ with $\mathrm{C} / \mathrm{W} \in F(i)$.

Theorem 4.1. Given a suitable SDML determined by $(I, \preccurlyeq, F)$, the linear nested system $\mathrm{LNS}_{(I, \preccurlyeq, F)}$ is correct and cut-free complete w.r.t. the sequent system $\mathrm{G}_{(I, \leqslant, F)}$.

Proof. For correctness, we translate a $\mathrm{LNS}_{(I, \preccurlyeq, F)}$ derivation into a $\mathrm{G}_{(I, \preccurlyeq, F)}$ derivation, discarding everything apart from the last component of the linear nested sequents, and translating blocks of modal rules into the corresponding modal sequent rules. For example, consider a block of proof in $\operatorname{LNS}_{(I, \leqslant, F)}$ consisting of an application of $!^{i}$ followed by $n$ applications of $?^{i}$ and an application of $r$ (bottom-up). This is translated into an application of the rule $\mathrm{K}_{i}$ in $\mathrm{G}_{(I, \leqslant, F)}$. Similarly for the rules for $\mathrm{T}$.

For completeness, we again simulate the sequent rules in the last components, as in Theorem 2.1.

We devote the rest of this section to showing how to specify, in a natural way, LNS $_{(I, \leqslant, F)}$ into LL. This could be seen just as a curious result and/or an extension of a series of works on using linear logic as 
a framework for specifying logical systems (see e.g. [MP13, NPR14]). But it is, in fact, an important result for at least two reasons: (1) it shows that SELL itself can be specified in linear logic; hence LL is more than ever universal, in the sense that it carries itself all the information of its extensions; and (2) it suggests that the difficulty of specifying a certain logical system in linear logic can mean that sequent systems may not be the best framework for describing that particular logic. For instance, while the usual sequent system for S4 cannot be naturally specified into LL, variations of it using labels [NvP11] or linear nested sequents [Lel15] have a natural and direct specification in LL (see [NPR14, LP15]). This suggests, again, the universality flavor of linear logic.

For encoding $\mathrm{LNS}_{(I, \leqslant, F)}$ into LL we need to describe the LNS structure in the language of LL. For this we first transform a LNS into its labeled correspondent (see also [LP15]).

\subsection{Labeled line sequent systems}

As pointed out in Section 2.2, being able to restrict linear nested sequents to their end-active version (see Definition 3) makes it possible to propose adequate labeled versions for such systems.

Let SV a countable infinite set of state variables (denoted by $x, y, z, \ldots$ ), disjoint from the set of propositional variables. A labeled formula has the form $x: A$ where $x \in \mathrm{SV}$ and $A$ is a formula. If $\Gamma=\left\{A_{1}, \ldots, A_{n}\right\}$ is a multiset of formulae, then $x: \Gamma$ denotes the multiset $\left\{x: A_{1}, \ldots, x: A_{n}\right\}$ of labelled formulas.

Definition 8. Let $\mathcal{R}$ be a relation set, that is, a set of relation terms of the form $x R y$ for some relation symbol $R$. A labeled line sequent LLS is a labeled sequent $\mathcal{R}, X$ where

1. $\mathcal{R}$ is a singleton;

2. $X$ is a multiset of formulas of the shape $x: F$ where $x$ is a state variable and $F$ is a formula

3. every state variable $x$ that occurs in $\mathcal{R}$ must also occur in $X$.

A labeled line sequent calculus is a labeled sequent calculus whose initial sequents and inference rules are constructed from LLS.

It is straightforward to construct a LLS inference rule from an inference rule of an end-active LNS calculus. The procedure, which can be automated, is the same as the one presented in [GR12, LP15, LP17], the only difference being that the different nesting operators considered here give rise to different relation symbols. We illustrate it in the next example for the special case of only one nesting operator.

Example 4.1. For a state variable $x$, define the mapping $\mathbb{T L}_{x}$ from $\mathrm{LNS}$ to end-active $\mathrm{LLS}$ as follows

$$
\begin{array}{lll}
\operatorname{TLL}_{x_{1}}\left(\Gamma_{1}\right) & =x_{0} R x_{1}, x_{1}: \Gamma_{1} \\
\operatorname{TLL}_{x_{n}}\left(\Gamma_{1} / / \ldots / / \Gamma_{n}\right) & =x_{n-1} R x_{n}, x_{1}: \Gamma_{1}, \ldots, x_{n}: \Gamma_{n} \quad n>1
\end{array}
$$

with all state variables pairwise distinct. Consider the following application of the rule !:

$$
\frac{\Gamma_{1} / / \ldots / / \Gamma_{n-1} / / \Gamma_{n} \| A}{\Gamma_{1} / / \ldots / / \Gamma_{n-1} / / \Gamma_{n}, ! A} !
$$

Applying $\mathbb{T L}_{x}$ to the conclusion we obtain $x_{n-1} R x_{n}, X, x_{n}: ! A$, where $X=x_{1}: \Gamma_{1}, \ldots, x_{n}: \Gamma_{n}$. Applying $\mathbb{T L}_{x}$ to the premise we obtain $x_{n} R_{\mathrm{r}} x_{n+1}, X, x_{n+1}: A$, where $R_{\mathrm{r}}$ is the relation corresponding to $\|$. We thus obtain an application of the LLS rule

$$
\frac{x_{n} R_{\mathrm{r}} x_{n+1}, X, x_{n+1}: A}{x_{n-1} R x_{n}, X, x_{n}: ! A} \mathbb{T L}_{x}(!)
$$




\begin{tabular}{|c|c|c|c|c|}
\hline & $\frac{x R_{\mathrm{r}}^{i} y, X, y: F}{x R_{\mathrm{r}}^{i} y, X, x: ?^{j} F}$ & $?^{i}{ }_{\mathrm{k}}($ for $j \in \uparrow(i))$ & $\frac{y R_{\mathrm{r}}^{i} z, X, z: F}{x R^{k} y, X, y: !^{i} F} !^{i}$ & $\frac{x R^{i} y, X}{x R_{\mathrm{r}}^{i} y, X} \mathrm{r}$ \\
\hline$\frac{x R_{\mathrm{r}}^{i} y, X, y: ?^{j} F}{x R_{r}^{i} y, X, x: ?^{j} F}$ & $?^{i}{ }_{4}\left(\right.$ for $\left.j \in \uparrow^{4}(i)\right)$ & $\frac{y R_{\mathrm{r}}^{i} z, X, z: F}{x R^{k} y, X, y: ?^{i} F}$ & $?^{i}{ }_{d}($ for $\mathrm{D} \in F(i))$ & $\frac{x R^{k} y, X, y: F}{x R^{k} y, X, y: ?^{i} F} ?_{\mathrm{t}}^{i}($ for $\mathrm{T} \in F(i))$ \\
\hline
\end{tabular}

Figure 8: Labeled line sequent calculus $\operatorname{LLS}_{(I, \leqslant, F)} . z$ is a fresh variable in rules $?_{\mathrm{d}}^{i}$ and $!^{i}$.

In the general case with more than one nesting operator we will denote by $R^{i}$ the relation corresponding to $/{ }^{i}$, by $R_{\mathrm{r}}^{i}$ the relation corresponding to $\|^{i}$, and by $R_{\mathrm{p}}$ the one corresponding to $\|_{\mathrm{p}}$. Figure 8 presents the modal rules for the labeled line calculus $\operatorname{LLS}_{(I, \leqslant, F)}$. By construction it is straightforward to see that the calculus is correct and complete.

\subsection{Specifying $\operatorname{LNS}_{(I, \leqslant, F)}$ in linear logic}

In [MP13] classical linear logic was used as the logical framework for specifying a number of logical and computational systems. The idea is simple: use a meta-level predicate $\lceil\cdot\rceil$ for identifying objects that appear in the sequents in the object logic. Hence, object-level sequents of the form $A_{1}, \ldots, A_{n}$ (where $n \geq 1$ ) are specified as the multiset $\left\lceil A_{1}\right\rceil, \ldots,\left\lceil A_{m}\right\rceil$.

Inference rules are specified by a rewriting clause that replaces the active formula in the conclusion by the active formulas in the premises. The linear logic connectives indicate how these object level formulas are connected: contexts are copied $(\&)$ or split $(\otimes)$, in different inference rules $(\oplus)$ or in the same sequent (४). As a matter of example, the additive versions of the inference rules for disjunction and conjunction in a single-sided sequent calculus for classical logic

$$
\frac{\Gamma, A}{\Gamma, A \vee B} \vee_{1} \quad \frac{\Gamma, B}{\Gamma, A \vee B} \vee_{2} \quad \frac{\Gamma, A \quad \Gamma, B}{\Gamma, A \wedge B} \wedge
$$

are specified as

$$
\vee: \exists A, B .\left(\lceil A \vee B\rceil^{\perp} \otimes(\lceil A\rceil \oplus\lceil B\rceil)\right) \quad \wedge: \exists A, B .\left(\lceil A \wedge B\rceil^{\perp} \otimes(\lceil A\rceil \&\lceil B\rceil)\right)
$$

The non-locality of the standard sequent rules for modal logic rendered this approach not directly suitable for encoding calculi for modal logics. This problem is avoided in the LNS calculi. The encoding of modal rules into LL is depicted in Figure 9, while the encoding of MALL connectives can be found in Figure 10. Observe that all clauses are implicitly existentially quantified. Object-level linear logic is specified reusing the same symbols that appear at the meta-level, namely, $\otimes, \diamond, \perp, 1, \&, \oplus, \top, \forall, \exists$ and negation $(\cdot)^{\perp}$ for atoms.

We assume that all $L L$ atomic predicates have negative polarity.

Note that if $I$ contains infinitely many indices, then the specification of $\operatorname{LLS}_{(I, \leqslant, F)}$ may contain infinitely many clauses, one for each $j \in I, j \in \uparrow(i)$ and/or $j \in \uparrow^{4}(i)$. This is not a problem, however, since by the subformula property of $\mathrm{G}_{(I, \ll, F)}$, rules mentioning modalities not occurring in a sequent $\Gamma$ do not occur in a derivation of $\Gamma$.

The following theorem shows that, in fact, the specification of modal rules into clauses in LL is, correct. The proof is similar to the one in [LP15].

Theorem 4.2 (Adequacy). The specification of the linear nested modal rules in Figure 8 into the LL clauses given in Figure 9 is adequate in the sense that a focused step in LL over a clause corresponds exactly to the application of the respective linear nested modal rule. 


$\begin{array}{ll}\left(?^{i}{ }_{\mathrm{k}}\right) & \left\lceil x: ?^{j} F\right\rceil^{\perp} \otimes R_{\mathrm{r}}^{i}(x, y)^{\perp} \otimes\left(\lceil y: F\rceil 8 R_{\mathrm{r}}^{i}(x, y)\right) \\ \left(?^{i}{ }_{4}\right) & \left\lceil x: ?^{j} F\right\rceil^{\perp} \otimes R_{\mathrm{r}}^{i}(x, y)^{\perp} \otimes\left(\left\lceil y: ?^{i} F\right\rceil 8 R_{\mathrm{r}}^{i}(x, y)\right) \\ \left(?^{i}{ }_{\mathrm{d}}\right) & \left\lceil y: ?^{i} F\right\rceil^{\perp} \otimes R^{k}(x, y)^{\perp} \otimes \forall z .\left(\lceil z: F\rceil 8 R_{\mathrm{r}}^{i}(y, z)\right) \\ \left(?^{i}\right) & \left\lceil y: ?^{i} F\right\rceil^{\perp} \otimes R^{k}(x, y)^{\perp} \otimes\left(\lceil y: F\rceil 8 R^{k}(x, y)\right) \\ \left(!^{i}\right) & \left\lceil y: !^{i} F\right\rceil^{\perp} \otimes R^{k}(x, y)^{\perp} \otimes \forall z .\left(\lceil z: F\rceil 8 R_{\mathrm{r}}^{i}(y, z)\right) \\ (\mathrm{r}) & R_{\mathrm{r}}^{i}(x, y)^{\perp} \otimes R^{i}(x, y)\end{array}$

Figure 9: Specification of $\operatorname{LLS}_{(I, \leqslant, F)}$ as clauses in LL. All the variables are bounded by an outermost existential quantifier.

\begin{tabular}{ll}
\hline$(\otimes)$ & $\lceil x: A \otimes B\rceil^{\perp} \otimes R(z, x)^{\perp} \otimes(\lceil x: A\rceil 8 R(z, x)) \otimes(\lceil x: B\rceil 8 R(z, x))$. \\
$(\&)$ & $\lceil x: A \& B\rceil^{\perp} \otimes R(z, x)^{\perp} \otimes(\lceil x: A\rceil \&\lceil x: B\rceil) 8 R(z, x)$. \\
$(\oplus)$ & $\lceil x: A \oplus B\rceil^{\perp} \otimes R(z, x)^{\perp} \otimes(\lceil x: A\rceil 8 R(z, x)) \oplus(\lceil x: B\rceil 8 R(z, x))$. \\
$(8)$ & $\lceil x: A 8 B\rceil^{\perp} \otimes R(z, x)^{\perp} \otimes\lceil x: A\rceil 8\lceil x: B\rceil 8 R(z, x)$. \\
$(\forall)$ & $\lceil x: \forall B\rceil^{\perp} \otimes R(z, x)^{\perp} \otimes \forall w .(\lceil x: B w\rceil 8 R(z, x))$. \\
$(\exists)$ & $\lceil x: \exists B\rceil^{\perp} \otimes R(z, x)^{\perp} \otimes \exists w .(\lceil x: B w\rceil 8 R(z, x))$. \\
$(1)$ & $\lceil x: 1\rceil^{\perp} \otimes R(z, x)^{\perp} \otimes 1$. \\
$(\perp)$ & $\lceil x: \perp\rceil^{\perp} \otimes R(z, x)^{\perp} \otimes R(z, x)$. \\
(pt) & $\lceil x: \top\rceil^{\perp} \otimes R(z, x)^{\perp} \otimes \forall y .\left(\lceil y: \top\rceil 8 R_{\mathrm{p}}(x, y)\right)$. \\
(tw) & $\lceil x: A\rceil^{\perp} \otimes R_{\mathrm{p}}(x, y)^{\perp} \otimes R_{\mathrm{p}}(x, y)$. \\
(T) & $\lceil y: \top\rceil^{\perp} \otimes R_{\mathrm{p}}(x, y)^{\perp} \otimes 1$. \\
\hline
\end{tabular}

Figure 10: Specification of $L N S_{\text {MALL }}$ in linear logic.

Observe that this result implies that (the linear nested version of) SELL can be encoded in linear logic. While this may come as a surprise, it only means that formulas in SELL marked with subexponentials are, in fact, suitable labelled linear logic formulas. More precisely, the rules in $L N S_{L L}$ provide a finer mechanism that allows us to handle, inside LL, the SELL promotion rule: we control, one by one, the formulas that can be promoted. We can thus mimic both the compartmentalization of the context in SELL (due to the subexponentials) as well as its promotion rule.

\subsection{Universal theorem prover for linear modalities}

We implemented in Maude (http://maude.cs.illinois.edu) a prototypical version of the endactive, focused version of the linear nested rules in Figure 7. The prover is parametric in the underlying multimodal systems. Hence, it is possible to specify signatures defining the set of indexes $I$ as well as their logical behavior $\left(\mathrm{LL}, \mathrm{LL}_{b}\right)$, modalities $(\mathrm{K}, 4, \mathrm{D}, \mathrm{T})$ and interaction axioms (see Definition 4).

The source files can be found at http://subsell .logic.at/SDML/. In that URL, the reader may find also a web-based version of the system for some predefined instances of SDML.

Sequents in the prover have two different shapes, unfocused $\left[\Gamma_{U}\right][\Gamma] \Delta ; \Delta^{\prime}$ and focused sequents $\Downarrow F \quad\left[\Gamma_{U}\right][\Gamma] \Delta ; \Delta^{\prime}$. The context $\Gamma_{U}$ stores all the formulas marked with the modality ? ${ }^{s}$ whenever $\mathrm{W}, \mathrm{C} \in F(s)$. In other case, a formula of the shape ? $?^{s} F$ is stored into the $\Gamma$ context where those structural axioms are not allowed, i.e, $s$ is a bounded exponential $\left(\mathrm{LL}_{b}\right)$. A third context, storing affine exponentials $\left(\mathrm{LL}_{a}\right)$ (with only W), may also be added. $\Delta^{\prime}$ is the general linear context and $\Delta$ stores positive and atomic formulas that cannot be introduced in a unfocus phase.

The invertible rules were implemented as part of an equational theory $\left[\mathrm{CDE}^{+} 07\right]$, thus avoiding unnecessary branching in the proof search procedure. Roughly speaking, before applying a positive rule, 
the prover performs the simplifications dictated by the equational theory.

Besides the negative rules in Figure 4, we also added the following rule for the modality 4:

$$
\frac{\left[\Gamma_{U}^{1}, j: \Psi\right]\left[\Gamma^{1}\right] \Delta^{1} ; \Delta^{\prime 1} / / /^{i}\left[\Gamma_{U}^{2} \cup j: \Psi\right]\left[\Gamma^{2}\right] \Delta^{2} ; \Delta^{\prime 2}}{\left[\Gamma_{U}^{1}, j: \Psi\right]\left[\Gamma^{1}\right] \Delta^{1} ; \Delta^{\prime 1} / /^{i}\left[\Gamma_{U}^{2}\right]\left[\Gamma^{2}\right] \Delta^{2} ; \Delta^{\prime 2}}
$$

whenever $i \leq j$ and $4 \in F(j)$. Note that this is a safe simplification when $\mathrm{W}, \mathrm{C} \in F(j)$.

The non-invertible rules, as expected, were specified as Maude's rewriting rules.

The search facilities in Maude can be used to perform some experiments in proving formulas pertaining to different logics by simply setting the parameter $(I, \leqslant, F)$. For instance, we got for free a prover for SELL. We have also proved canonical examples of modal logics adopted to the linear setting described in this paper. The experiments can be found on the site of the implementation.

\section{Concluding remarks and related/future work}

This paper has three principal results: (1) to propose a uniform presentation to linear logics featuring different axioms of modalities; (2) to build theorem provers for different logics, based on the same grounds and parametric in the modal/structural axioms; and (3) to allow for the use of the same logical framework for reasoning about all such logical systems.

Since all these goals strongly depend on modular proof systems for substructural/modal systems, our starting point was to formulate a local system for linear logic $\left(L N S_{L L}\right)$, since locality often enables modularity. The linear nested sequent system $\mathrm{LNS}_{\mathrm{LL}}$ can be seen as an adaptation of the 2-sequent calculus for linear logic presented in [GMM98]. Amazingly enough, the series of works on modalities and 2-sequents [Mas92, GMM98] received little attention until the work in [Lel15], where it was noticed that 2-sequents can be viewed as a restriction of nested sequents.

However, while in [GMM98] the focus was on elementary and light modalities in linear logic, in this paper we generalize, in a non-trivial way, the notion of (multi) modalities in LL. This includes ELL and, while we do not deal with the light modal operator explicitly, it could be easily added to our approach, following the same lines as in [GMM98].

It turns out that multi-modalities are often added to linear logic by defining whole algebraic structures, that are then attached to the logical system via an exponential signature. In this paper, we have chosen a rather different approach: add dependencies between (possible different) logics, so that the algebraic structure is determined by such dependencies. This elegant and modular way of presenting exponentials serves as a starting point for proposing different modalities for different logics. For example, on changing the base logic from classical to intuitionistic linear logic, one can talk about (multi) modalities over constructive logics (like Lambek Calculus with exchange, for instance).

Moreover, by restricting the set of modal axioms, it is possible to extend the definition of subexponentials so to have other modal/structural behaviors, other than just being bounded/unbounded. This should contribute, for example, to the development of new (declarative) constructs for process calculi along the same lines as done in [OPN15].

Another interesting line of research to be pursued is to characterize certain object level properties at the meta level. In [MP13], LL was used to give sufficient conditions to guarantee admissibility of the cut rule and/or atomic initial axiom in several object level logics. This result is rather elegant, in the sense that it is parametric in the object logic. At the same time, it seems weak since it depends on an adequate specification of that logic in LL. While some sequent systems require subexponentials for guaranteeing the adequacy of the specification [NPR14], others cannot be specified at all in a natural way (e.g.non-commutative or focused systems). In this paper we showed that all the specifications done in SELL can be translated to LL (since SELL itself can be specified in LL). Moreover, in [LP15] we 
presented end-active LNS systems for a class of modal logics, and those systems can be adequately specified in LL. Hence, while we have enlarged the number of systems that can be specified, we changed their logical structure (from sequent to linear nested systems). This means that the conditions presented in [MP13] may not be valid for characterizing the object level properties anymore. These conditions depend strongly on mimicking, at the meta-level, the cut-elimination process at the object level. Hence, one research direction would be to analyze the behavior of cut-elimination for end-active linear nested systems and see if this can be captured in LL.

Still about the encodings, it is worth noticing that the choice of LL as the meta-level framework is one among many possible. The important aspect here is that the resulting specification clauses are bipoles, that is, formulas that contain no positive connectives in the scope of negative ones. In this way, focusing can be used to guarantee the adequacy of the specification, in the sense that one focused step in the meta-level corresponds exactly to the application of the specified rule at the object level. This means that our method is general enough and can be adapted to other logical frameworks [MV15, MMV16]. 


\section{References}

[Ach16] Antonis Achilleos. Modal logics with hard diamond-free fragments. In Sergei Artemov and Anil Nerode, editors, LFCS 2016, volume 9537 of LNCS, pages 1-13. Springer International Publishing, 2016.

[And92] Jean-Marc Andreoli. Logic programming with focusing proofs in linear logic. Journal of Logic and Computation, 2(3):297-347, 1992.

[Bal00] Matteo Baldoni. Normal Multimodal Logics with Interaction Axioms, pages 33-57. Springer Netherlands, Dordrecht, 2000.

[Brü09] Kai Brünnler. Deep sequent systems for modal logic. Arch. Math. Log., 48:551-577, 2009.

[CDE ${ }^{+}$07] Manuel Clavel, Francisco Durán, Steven Eker, Patrick Lincoln, Narciso Martí-Oliet, José Meseguer, and Carolyn L. Talcott, editors. All About Maude - A High-Performance Logical Framework, How to Specify, Program and Verify Systems in Rewriting Logic, volume 4350 of Lecture Notes in Computer Science. Springer, 2007.

[CMS16] Kaustuv Chaudhuri, Sonia Marin, and Lutz Straßburger. Focused and synthetic nested sequents. In Bart Jacobs and Christof Löding, editors, Proc. of FOSSACS 2016., volume 9634 of Lecture Notes in Computer Science, pages 390-407. Springer, 2016.

[Dem00] Stéphane Demri. Complexity of simple dependent bimodal logics. In Roy Dyckhoff, editor, TABLEAUX 2000, volume 1847 of LNCS, pages 190-204. Springer, 2000.

[DFMV15] Martin Davis, Ansgar Fehnker, Annabelle McIver, and Andrei Voronkov, editors. Logic for Programming, Artificial Intelligence, and Reasoning - 20th International Conference, LPAR-20 2015, Suva, Fiji, November 24-28, 2015, Proceedings, volume 9450 of Lecture Notes in Computer Science. Springer, 2015.

[DJS93] Vincent Danos, Jean-Baptiste Joinet, and Harold Schellinx. The structure of exponentials: Uncovering the dynamics of linear logic proofs. In Georg Gottlob, Alexander Leitsch, and Daniele Mundici, editors, Kurt Gödel Colloquium, volume 713 of LNCS, pages 159-171. Springer, 1993.

[Gir87] Jean-Yves Girard. Linear logic. Theoretical Computer Science, 50:1-102, 1987.

[Gir98] Jean-Yves Girard. Light linear logic. Information and Computation, 143(2):175-204, 1998.

[GMM98] Stefano Guerrini, Simone Martini, and Andrea Masini. An analysis of (linear) exponentials based on extended sequents. Logic Journal of the IGPL, 6(5):735-753, 1998.

[GR12] Rajeev Goré and Revantha Ramanayake. Labelled tree sequents, tree hypersequents and nested (deep) sequents. In Thomas Bolander, Torben Braüner, Silvio Ghilardi, and Lawrence S. Moss, editors, Advances in Modal Logic 9, papers from the ninth conference on "Advances in Modal Logic," held in Copenhagen, Denmark, 22-25 August 2012, pages 279-299. College Publications, 2012.

[Gug07] Alessio Guglielmi. A system of interaction and structure. ACM Trans. on Computational Logic, 8(1):1-64, January 2007.

[Lel13] Björn Lellmann. Sequent Calculi with Context Restrictions and Applications to Conditional Logic. PhD thesis, Imperial College London, 2013.

[Lel15] Björn Lellmann. Linear nested sequents, 2-sequents and hypersequents. In Hans De Nivelle, editor, Automated Reasoning with Analytic Tableaux and Related Methods - 24th International Conference, TABLEAUX 2015, Wrocław, Poland, September 21-24, 2015. Proceedings, volume 9323 of Lecture Notes in Computer Science, pages 135-150. Springer, 2015.

[LP13] Björn Lellmann and Dirk Pattinson. Constructing cut free sequent systems with context restrictions based on classical or intuitionistic logic. In ICLA 2013, volume 7750 of LNCS, pages 148-160. Springer, 2013.

[LP15] Björn Lellmann and Elaine Pimentel. Proof search in nested sequent calculi. In Davis et al. [DFMV15], pages 558-574.

[LP17] Björn Lellmann and Elaine Pimentel. Modularisation of sequent calculi for normal and non-normal modalities. CoRR, abs/1702.08193, 2017. 
[Mas92] Andrea Masini. 2-sequent calculus: a proof theory of modalities. Ann. Pure Appl. Logic, 58:229-246, 1992.

[MM94] Simone Martini and Andrea Masini. A modal view of linear logic. Journal of Symbolic Logic, 59(3):888-899, 1994.

[MMV16] Sonia Marin, Dale Miller, and Marco Volpe. A focused framework for emulating modal proof systems. In AiML 16, 2016.

[MP02] Dale Miller and Elaine Pimentel. Using linear logic to reason about sequent systems. In Uwe Egly and Christian G. Fermüller, editors, International Conference on Automated Reasoning with Analytic Tableaux and Related Methods, volume 2381 of LNCS, pages 2-23. Springer, 2002.

[MP04] Dale Miller and Elaine Pimentel. Linear logic as a framework for specifying sequent calculus. In Jan van Eijck, Vincent van Oostrom, and Albert Visser, editors, Logic Colloquium '99: Proceedings of the Annual European Summer Meeting of the Association for Symbolic Logic, Lecture Notes in Logic, pages 111-135. A K Peters Ltd, 2004.

[MP13] Dale Miller and Elaine Pimentel. A formal framework for specifying sequent calculus proof systems. Theoretical Computer Science, 474:98-116, 2013.

[MV15] Dale Miller and Marco Volpe. Focused labeled proof systems for modal logic. In Davis et al. [DFMV15], pages 266-280.

[NM09] Vivek Nigam and Dale Miller. Algorithmic specifications in linear logic with subexponentials. In $A C M$ SIGPLAN Conference on Principles and Practice of Declarative Programming (PPDP), pages 129-140, 2009.

[NOP13] Vivek Nigam, Carlos Olarte, and Elaine Pimentel. A general proof system for modalities in concurrent constraint programming. In CONCUR 2013 - Concurrency Theory - 24th International Conference, CONCUR 2013, Buenos Aires, Argentina, August 27-30, 2013. Proceedings, pages 410-424, 2013.

[NPR14] Vivek Nigam, Elaine Pimentel, and Giselle Reis. An extended framework for specifying and reasoning about proof systems. Journal of Logic and Computation, 26, 2014.

[NvP11] Sara Negri and Jan van Plato. Proof Analysis: A Contribution to Hilbert's Last Problem. Cambridge University Press, 2011.

[OPN15] Carlos Olarte, Elaine Pimentel, and Vivek Nigam. Subexponential concurrent constraint programming. Theoretical Computer Science, 606:98-120, 2015.

[PM05] Elaine Pimentel and Dale Miller. On the specification of sequent systems. In LPAR 2005: 12th International Conference on Logic for Programming, Artificial Intelligence and Reasoning, number 3835 in LNAI, pages 352-366, 2005.

[Pog09] Francesca Poggiolesi. The method of tree-hypersequents for modal propositional logic. In Towards Mathematical Philosophy, volume 28 of Trends In Logic, pages 31-51. Springer, 2009.

[PON14] Elaine Pimentel, Carlos Olarte, and Vivek Nigam. A proof theoretic study of soft concurrent constraint programming. TPLP, 14(4-5):649-663, 2014.

[Str02] Lutz Straßburger. A local system for linear logic. In Proceedings of LPAR 2002, number 2514 in LNCS, pages 388-402, January 2002.

[Tro92] Anne S. Troelstra. Lectures on Linear Logic. CSLI Lecture Notes 29, Center for the Study of Language and Information, Stanford, California, 1992.

[Vig00] Luca Viganò. Labelled non-classical logics. Kluwer, 2000. 